\title{
Impact of Problem-based Learning Strategy on Students' Mathematical Value among Secondary School Students
}

\author{
Fatimah Ramli ${ }^{1}$, Ahmad Fauzi Mohd Ayub ${ }^{1,2, *}$, Hutkemri Zulnaidi ${ }^{3}$, Nur Raidah Salim ${ }^{1}$, Kathiresan Gopal ${ }^{1}$ \\ ${ }^{1}$ Institute for Mathematical Research, Universiti Putra Malaysia, Malaysia \\ ${ }^{2}$ Department of Foundation Education, Faculty of Educational Studies, Universiti Putra Malaysia, Malaysia \\ ${ }^{3}$ Department of Mathematics and Science Education, Faculty of Education, University of Malaya, Malaysia
}

Received July 18, 2019; Revised May 30, 2020; Accepted June 4, 2020

Copyright $\mathrm{C} 2020$ by authors, all rights reserved. Authors agree that this article remains permanently open access under the terms of the Creative Commons Attribution License 4.0 International License

\begin{abstract}
This study examined the effectiveness of implementing a problem-based learning (PBL) strategy on the mathematical values of the mathematics secondary school. A quasi-experimental nonrandomized control group post-test design was conducted, which consisted of 62 students in two intact groups. 35 students were placed in the experimental group, while 27 students in the control group participated in this study. Students in the experimental group received the PBL instruction strategy, while the control group learned mathematics using conventional instructional in a classroom for eight weeks. Students' mathematical values were measured using a set of rubrics consisting of nine mathematics educational values. The results of this study showed the students from the PBL strategy group demonstrated significantly higher scores than the conventional instruction strategy group in the overall mathematical values and the subscales of accuracy, conjecturing, consistency, creativity, effective organization, efficient working/ strategies, flexibility, persistence, and systematic working. Therefore, it is recommended that the use of the PBL strategy would help students to understand mathematical values better compared to conventional instruction.
\end{abstract}

Keywords Conventional Instructional Strategy, Mathematical Values, Problem-based Learning Strategy, Quasi-experimental, Secondary School Students

\section{Introduction}

Under the 10th Malaysian Plan (2011-2015), the education system was revised and revamped to improve and sustain students' outcomes. The mathematics curriculum was specifically designed to inculcate "mathematical values" among Malaysian learners. Values in Mathematics were defined as the ability to do mathematics, understand mathematical ideas, and apply mathematical knowledge and skills responsibly in daily life (Ministry of Education, Malaysia, 2011).

Mathematical principles are clearly expressed to the extent that the linkages between mathematical concepts and their utility or application to the immediate environment of the learners displayed a high degree of honesty (Bishop, 1988; Seah, 2013).Values are the most important elements of learning and teaching in mathematics (Seah, 2002; Ramli et al ., 2018). However, identifying values is not easy (Brown, 2001). According to Swadener and Soedjadi (1988), identifying values as a concept or an idea has always been difficult. When looking at the definition of values, this can be described as personal choices which consider values or significance of action or concept, and also that individuals as members of society embrace or follow general aimsTherefore, values are reflected in concepts or ideas about anything. Values can be classified into two groups: aesthetic and ethical. Aesthetic values are concepts of beauty, but ethical values are notions that can be demonstrated as bad or good.These values form the wholeness in education.

The values were planned, portrayed, or espoused in a teachers' pedagogical approach in the classroom (Lim \& Ernest 1997; Chin, et al. 2001). The values portrayed by teachers in the mathematics classroom are linked to their pedagogical approaches (Seah \& Bishop, 2002). Seah and Bishop (2002) identified values held by teachers as their "magnetization" of affective factors such as beliefs and attitudes, and their subsequent internalization into their respective affective-cognitive personal systems. According to Bishop, Clarkson, Fitz Simons, and Seah (2000), there is 
very little understanding about the values teachers are teaching in math classes, especially how their lessons establish those values in their students. In mathematics teaching, values are rarely included on the discussion

Mathematics is the most value-free of all school subjects, not only between teachers but among parents, university mathematicians, and employers, it is a widespread misunderstanding. Mathematical values are the ones that indicate mathematical awareness in nature. They are produced by mathematicians drawn from different cultures (Bishop et al., 1999).

Examples of mathematical principles are proving the Pythagorean theorem in three different ways, and their recognition (Seah \& Bishop, 2000).Culture is a powerful determiner of mathematical values. Research shows that the basic values of all societies weren't shared. So, mathematics teachers working in different cultures do not teach the same values, and although they teach the same curriculum (Bishop et al . 2000).

Problem-based learning (PBL) is a teaching strategy where learning activities evolve around over a real-life problem (Barrows, 1986). PBL is one of the active learning models that support flexibility and creativity in learning. PBL exists as a method of teaching premised upon ideals of constructivism and student-centered learning. PBL was designed to address the students' unwillingness to apply learned knowledge in real-life situations and to solve a problem. PBL's basic challenge is to make students who come face-to - face with conditions in their professions which might be relevant to normal situations.

Students are encouraged to investigate and create alternative solutions or problem-solving decisions (Goodman 2010). The approach offers a rich learning background for student, contributing to the anchoring of new mathematical insights into concrete problems and experiences (Hung, 2016). More importantly, they develop a better understanding of concepts and can apply knowledge learned to solve problems in real-world situations, which leads to positive effects on their motivation toward learning (Hung, 2016). When using PBL, teachers help students focus on seeking solutions to issues in a real-life setting, enabling them to understand the situation in which the problem occurs (Nasir, Hand \& Taylor, 2008)

By adding intervention in the teaching and learning process helped students to understand better and perform better scores in their performance scores. Research work by Amalia, Surya, and Syahputra (2017) indicated also that PBL strategy is beneficial in enhancing the students ' ability to overcome mathematics problems.

Meanwhile, the same strategy also found significance in learning Calculus in research conducted by Mokhtar, Tarmizi, Ayub, and Tarmizi. (2010). Two studies showed by using Higher-order thinking modules as intervention had helped students achieve a better score (Ibrahim et. al,
2019) and reduce pupils' errors compared to the traditional method in the Topic of Measurement and Geometry. Intervention by using technology module related also shown a positive result when integrating it in the classroom, Bakar et. al(2015) and Saha et. al (2010) studies had shown that by using GeoGebra Software as their intervention had helped students to achieve a better score.

\section{Research Objective}

The goal of this study was to investigate the effect on the topics of Pythagoras theorem, Transformation, Solid Geometry II, and statistics of using the PBL strategy on the mathematical value of students as compared to conventional instruction (CI) strategy. Mathematics Values contains the following nine educational principles in mathematics: precision, uncertainty, continuity, imagination, effective organization, productive work / strategies, versatility, patience, and systematic practice.

\section{Methodology}

\section{Research Design}

A research design includes the research strategies to get answers for the research or problems (Kumar, 2011). The research design for this study is the pre-post quasi-experimental design to investigate the effects of the PBL strategy on students' cognitive performance, mathematical values, and motivation in the learning of mathematics.This quasi-experiment of causal comparing is most ideal for investigating the effectiveness of an intact group intervention (teaching strategy) and it is used when the true experimental design is not possible (Creswell, 2014)

When there are at least two compare groups, the quasi-experimental design fulfills the criterion of a strong research methodology: one control groups and one treatment group (see Table 1).

Table 1. Non-equivalent control group design

\begin{tabular}{|c|c|c|c|}
\hline & Pre-test & Behaviors & Post-test \\
\hline Treatment & $\mathrm{O}_{1}$ & $X_{1}$ & $\mathrm{O}_{3}$ \\
\hline Control & $\mathrm{O} 2$ & $X_{2}$ & $\mathrm{O}_{4}$ \\
\hline
\end{tabular}

Notes:

$X_{1}$ : PBL strategy was applied

$X_{2}$ : Normal activities were used

$0_{1}$ : Pre-test on Treatment class

$0_{2}$ : Pre-test on Control class

: Post-test on Treatment class

$0_{4}^{3}$ : Post-test on Control class

The purpose of having different experimental and control groups is to control any confounding extraneous variables that may threaten the internal validity of the design. Two instructional strategies, the PBL strategy, and the CI strategy, were conducted in both phases of the study. 
For the experimental groups, the PBL strategy was implemented. It was a group instruction with the following activities: the mathematical concepts were explored by the students using the seven steps; clarifying the setting, defining the problems, analyzing and investigating the problem, restructuring the problem, formulating the learning goal, learning individually, and reporting back to the group (Hung, 2011). In the development phase, students were discussing and solving the mathematical problems in groups. At the end of the lesson, each group presented the findings. For the control groups, the CI strategy was implemented. It was a whole-class instruction, without incorporating the use of PBL instruction. The following activities were implemented, and the mathematical concepts were explained using only the whiteboard. The solving of the mathematical problems related to the concepts was explained. In the development phase, students were provided mathematical problems to solve individually, and subsequently, discussion of the problem and the conclusion of the lesson were handled by the teacher. At the end of the lesson, each student was given an evaluation where students were asked to solve the problem using the paper and pencil method only.

\section{Participants}

At a selected school in Negeri Sembilan 62 female students from two intact classes of Form Two participated. The randomly assigned groups were the PBL strategy group (35 students) and the CI group (27 students). The intervention class learned mathematics using the PBL instruction strategy, while the control group learned mathematics thru eight weeks with following their normal classroom instructional activities. Both groups received the same conditions regarding the content of the lessons, mathematical tasks and contact hours.

\section{Measurement}

The performance test was designed by the researcher to assess students' performance on the Pythagoras theorem, Transformation, and Solid Geometry II topics. The test inventory was distributed after the groups had received the treatment. Overall test performance consists of 12 items that were divided into Higher Order Thinking Skills (HOTS) (6 items) and Lower Order Thinking Skills (LOTS) (6 items) was prepared according to Bloom Taxonomy. This test was a systematic formal test, using a paper-and-pencil procedure and produced numerical scores. The scores for each problem solution were allotted one mark for each correct step utilized. All the questions were submitted to the validators. The validators agreed the achievement test was in line with the form two syllabus designed by the Curriculum Development Centre, Ministry of Education Malaysia (CDC). Some of the items used in this study are as follows.

1. State the number of surfaces for the following shapes

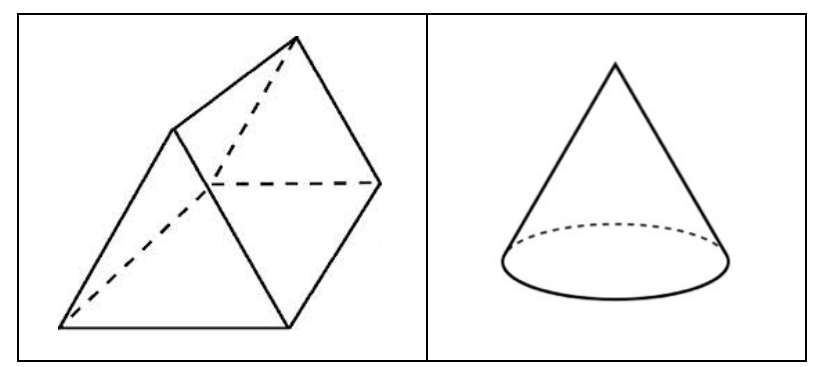

Number of spaces $=$

2. A milk tin made of stainless steel has a diameter of 10 $\mathrm{cm}$ and a height of $12 \mathrm{~cm}$.

a Calculate the amount of stainless steel needed to build a can of milk.

b Why can a tin manufacturer need to know the surface area of the milk can?

3. Jonathan was traveling from City A to the north for 5 $\mathrm{km}$ for a vacation in City A. Suddenly he received a phone call saying his grandmother was very ill. Jonathan continued traveling to the west for $7 \mathrm{~km}$ to his grandmother's house. What is the closest distance between his grandmother's home and Town A?

4. Figure 1 shows $Q^{\prime}$ is the image of $Q$ under $Z$ transformation $Z$. Describe in detail the transformation of Z. Justify it, 
y

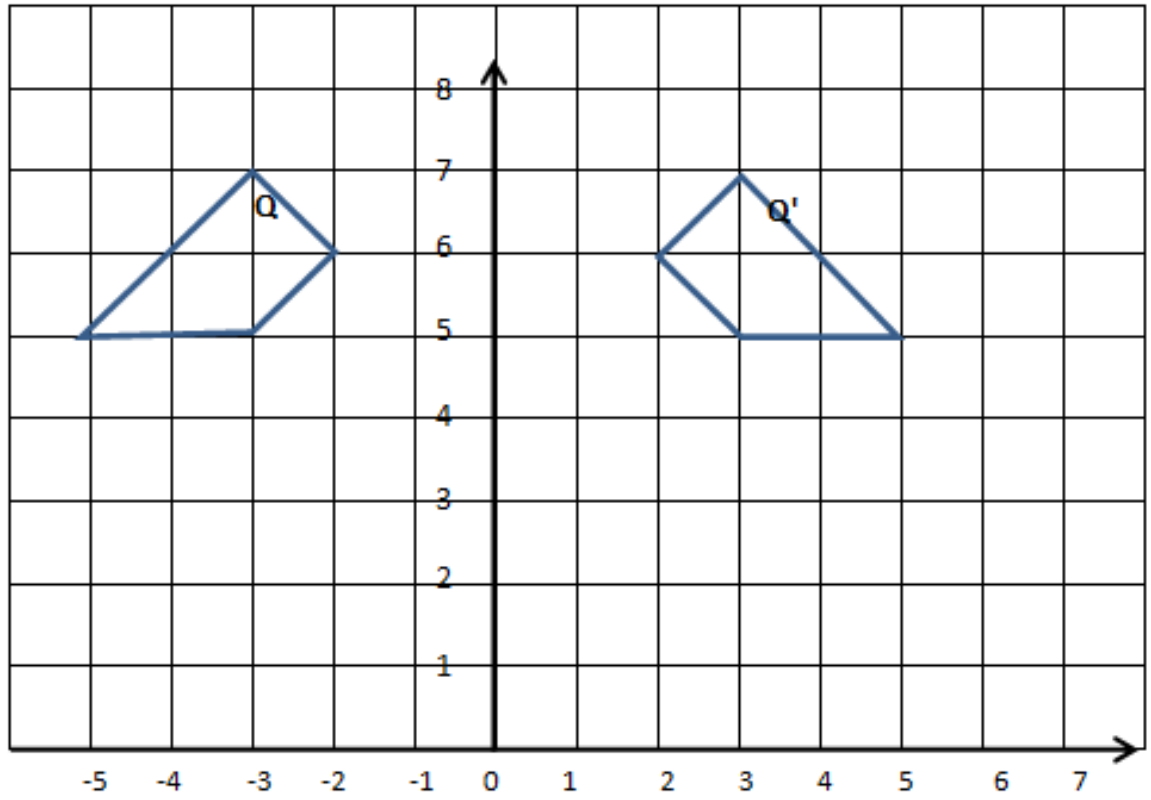

Figure 1. Transformation diagram of $Z$

Mathematical values are portrayed explicitly to the extent that the linkages between mathematical concepts and their usefulness or application to the learners' immediate environment exhibited a high degree of comprehensiveness (Bishop, 1988; Seah, 2013). These values were measured using the researcher-constructed rubric that employed the rubric scale and was expressed explicitly during problem-solving in terms of the nine mathematics educational values as stated above. Accuracy is defined as the definite idea of work and solution of the problem during the intervention of the problem-solving session based on students' mathematical value rubrics. Conjecturing is a form of hypothesis or conjecture that connects aspects of mathematics to a broader view of the problem, in which students can relate to a real-life situation. Consistency refers to the ability of the students to accurately interpret evidence, statements, graphics, and questions in the problem-solving session during the intervention. Creativity was defined as the ability of students to develop a logical, consistent plan to solve the problem, recognize the consequences of the solution, and articulate reasons for choosing solutions in problem-solving. An effective organization can be observed through the ability of students to organize and present mathematical ideas and information in a clear, systematic, effective, complete, and accurate manner during problem-solving. Efficient working strategies were observed through innovative strategies used by the students and their ability to make their corrections when needed. Flexibility is defined as the ability of the students to apply accurately multiple strategies to solve a problem during problem-solving. Systematic working refers to the students' ability to choose a plan and know exactly what to do to solve unfamiliar mathematics problems. These mathematical values were analyzed using students' problem-solving strategies. These values were measured using the researcher-constructed rubric that employed the rubric scale, which was expressed explicitly during problem-solving (see Table 2).

Table 2. Scoring criteria for determining mathematical values

\begin{tabular}{cl}
\hline Score & \multicolumn{1}{c}{ Mathematical values } \\
\hline 0 & No evidence \\
1 & All inappropriate \\
2 & Parts missing and/or contain errors \\
3 & Minor omissions or errors \\
4 & Appropriate and complete \\
\hline
\end{tabular}

\section{Pilot Study}

A pilot study was conducted by the researcher in May 2013. The pilot study was conducted to form two students from a public school in Hulu Langat district. They were not involved in the actual study but had a similar background to the actual sample. This pilot study was done to ascertain the aspects that contributed to the success of the actual experiment. The pilot study was conducted to validate the experimental procedures and process and to allow for familiarization of the experimental procedures to the researcher. Besides, during the pilot study, the researcher would establish the reliability of the instrument. The reliability of the items was analyzed using SPSS and Kuder Richardson formula 20 (KR-20). The reliability analysis of the performance test instrument using 
Kuder-Richardson Formula 20 (KR-20) was done to ensure the consistency of the instrument. After the pilot study, reliability coefficients for the performance test were calculated. The KR-20 reliability coefficient obtained from the pilot study was 0.73 for LOTS of questions and 0.75 for HOTs questions.

\section{Data Analysis}

The results were further analyzed using SPSS software version 25. One-way Multivariate analysis of variance (MANOVA) tests was conducted on the two groups to determine any differences between the students' mathematical value on the topics of the Pythagoras theorem, Transformation, Solid Geometry II, and statistics. Every individual in the sample was measured twice using a similar test before and after a period, and the two measurements data were compared. This quasi-experimental study did not involve a random selection of the respondents in either the treatment or control group. Hence, homogeneity testing was conducted to predetermine the intelligence profiles and levels of the thinking ability among samples. Therefore, a pre-test was conducted at the beginning of this study to determine the similarity of the two sample groups.

\section{Findings}

Students' Mathematical Values in the Learning of Mathematics by the PBL Strategy and CI Strategy Groups

A one-way MANOVA test was conducted to compare the students' mathematical values in the learning of mathematics by the PBL strategy and CI strategy groups during the problem-solving sessions (see Table 3). The analysis met the assumption of normality with skewness and kurtosis value in range -0.21 until -1.16 . The homogeneity of variance-covariance matrices, as calculated by Box's M test, is Box's $M=276.81, F=6.56$, $p=0.001$. The result indicated a significant difference existed in the mean of students' mathematical values in the learning of mathematics between the two groups between the PBL strategy group and the CI strategy group with Wilks' Lambda value $=0.265$, sig $=0.001(p<0.05)$ and partial eta square 0.735 . The magnitude of the differences demonstrated a considerably large effect (based on Cohen, 1988). This finding indicated that students who were exposed to the use of the PBL strategy achieved significantly higher on the overall mathematical values in the learning of mathematics compared to those who were taught using the CI strategy.

Table 3. One-way MANOVA test on students' mathematical values in the learning of mathematics by the PBL strategy and CI strategy groups

\begin{tabular}{|c|c|c|c|c|c|c|}
\hline Dependent Variable & Type III Sum of Squares & df & Mean Square & $F$ & Sig & Partial Eta Squared \\
\hline Accuracy & 34.314 & 1 & 34.314 & 84.145 & 0.001 & 0.584 \\
\hline Conjecturing & 26.281 & 1 & 26.281 & 87.777 & 0.001 & 0.594 \\
\hline Consistency & 24.537 & 1 & 24.537 & 77.921 & 0.001 & 0.565 \\
\hline Creativity & 14.498 & 1 & 14.498 & 75.344 & 0.001 & 0.557 \\
\hline Effective & 23.454 & 1 & 23.454 & 76.338 & 0.001 & 0.560 \\
\hline Efficient & 16.455 & 1 & 16.455 & 59.769 & 0.001 & 0.499 \\
\hline Flexibility & 12.132 & 1 & 12.132 & 75.664 & 0.001 & 0.558 \\
\hline Systematic & 23.622 & 1 & 23.622 & 88.499 & 0.001 & 0.596 \\
\hline
\end{tabular}


Table 4. Mean difference in students' mathematical values in the learning of mathematics by the PBL strategy and CI strategy groups

\begin{tabular}{|c|c|c|c|c|c|}
\hline \multirow{2}{*}{ Dependent Variable } & \multirow{2}{*}{ Group } & \multirow{2}{*}{ Mean } & \multirow{2}{*}{ Std. Error } & \multicolumn{2}{|c|}{$95 \%$ Confidence Interval } \\
\hline & & & & Lower Bound & Upper Bound \\
\hline \multirow{2}{*}{ Accuracy } & Treatment & 2.81 & 0.11 & 2.59 & 3.02 \\
\hline & Control & 1.31 & 0.12 & 1.06 & 1.55 \\
\hline \multirow{2}{*}{ Conjecturing } & Treatment & 2.24 & 0.09 & 2.05 & 2.42 \\
\hline & Control & 0.93 & 0.11 & 0.72 & 1.14 \\
\hline \multirow{2}{*}{ Consistency } & Treatment & 2.34 & 0.10 & 2.15 & 2.53 \\
\hline & Control & 1.07 & 0.11 & 0.86 & 1.29 \\
\hline \multirow{2}{*}{ Creativity } & Treatment & 1.67 & 0.07 & 1.52 & 1.82 \\
\hline & Control & 0.69 & 0.08 & 0.52 & 0.86 \\
\hline \multirow{2}{*}{ Effective } & Treatment & 2.19 & 0.09 & 2.00 & 2.38 \\
\hline & Control & 0.95 & 0.11 & 0.74 & 1.16 \\
\hline \multirow{2}{*}{ Efficient } & Treatment & 2.08 & 0.09 & 1.90 & 2.25 \\
\hline & Control & 1.04 & 0.10 & 0.84 & 1.24 \\
\hline \multirow{2}{*}{ Flexibility } & Treatment & 1.66 & 0.07 & 1.52 & 1.79 \\
\hline & Control & 0.77 & 0.08 & 0.61 & 0.92 \\
\hline \multirow{2}{*}{ Systematic } & Treatment & 2.13 & 0.09 & 1.96 & 2.31 \\
\hline & Control & 0.89 & 0.10 & 0.69 & 1.09 \\
\hline
\end{tabular}

There was a significant difference in accuracy $[F=$ 84.145 , sig $=0.001,(p<0.05)]$, conjecturing $[F=87.777$, $\operatorname{sig}=0.001,(p<0.05)]$, consistency $[F=77.921$, sig $=$ $0.001,(p<0.05)]$, creativity $[F=75.344, \operatorname{sig}=0.001,(p<$ $0.05)]$, effective $[F=76.338$, sig $=0.001,(p<0.05)]$, efficient $[F=59.769, \operatorname{sig}=0.001,(p<0.05)]$, flexibility $[F$ $=75.664$, sig $=0.001,(p<0.05)]$, and systematic $[F=$ 88.499 , sig $=0.001,(p<0.05)]$. The overall mean scores of the PBL strategy group were higher than the CI strategy group (See Table 4). The magnitude of the differences was considered large (0.499-0.596), based on Cohen (1988). This result revealed that students exposed to the use of the PBL strategy obtained substantially higher accuracy in mathematics learning as opposed to those taught using the CI strategy.

\section{Discussion}

Values in mathematics education are a relatively new area of research. A value is a standard thing regarded to exhibit the worth principle by which we live, a standard by which we judge what is important, and something that we aim for qualities to which we confront (Seah et al., 2001). Mathematics can never be context and value-free. Choosing suitable activities allows us to address these issues but also to see the relevance of mathematics within the wider dimension both as a tool for everyday life and as a creative discipline in its own right. Our teaching brings with it a set of theories on how children learn mathematics, and with our theories comes the potential for influencing students' beliefs about mathematics itself (Jones, 1999).
The ability to think mathematically and to use mathematical thinking to solve problems is an important goal in schooling. In this respect, mathematics attainment among learners will support science, technology, economic life, and development in a nation's economy.

This finding indicated that by using PBL on the topics of Pythagoras theorem, Transformation, and Solid Geometry II, the real-life problem in PBL instruction strategy induced the conjecturing values, in which students can relate with a real-life situation and form of hypothesis or conjecture that connects aspects of mathematics to a broader view of the problem. The activities in organizing the problem enhance the accuracy values. Self-directed learning allows students to determine their formulation of the problem within given subject area guidelines and helps the students to consistently and accurately interpret evidence, statements, graphics, and questions in the problem-solving session during the intervention. Experience learning in PBL strategies provides the opportunities for the students to build their own experiences and interests, which enhances the creativity values as students can develop a logical and consistent plan to solve problems, recognize consequences of solutions, and articulate reasons for choosing solutions in problem-solving. Systematic working refers to the students' ability to choose a plan and know exactly what to do to solve unfamiliar mathematics problems.

The PBL learning process, which requires the activities involving research, decision making, and writing, allows students to acquire deeper learning. These activities can induce flexibility values as students can apply accurately multiple strategies to solve a problem during problem-solving. Exemplary practice-students require the 
ability to transfer knowledge, theory, and methods from previously learned area to new ones. An effective organization can be observed through the ability of students to organize and present mathematical ideas and information in a clear, systematic, effective, complete, and accurate manner during problem-solving. PBL strategies involved inter-disciplinary learning where the problem can extend beyond traditional subject-related boundaries and methods. This shows that PBL strategies can enhance student working efficiency through innovative strategies used by the students and their ability to make their corrections when needed. Therefore, PBL instruction can be beneficial for students, as this instructional strategy has proven to improve students' mathematical values.

The study results provide implications for teachers to use the PBL strategy in classrooms. The use of the PBL strategy will improve the students' mathematical values. This PBL strategy also was recommended to teachers of other subjects for their lessons. However, further studies are required on the effects of the PBL strategy on other mathematical aspects, such as critical, creative, and reflective thinking skills. The reason is that the PBL strategy allows students to think critically, creatively, and reflectively in the process of discovering new ways of solving mathematical problems.

\section{Conclusions}

This study showed the potential and effect of the PBL strategy on students' mathematical values of the Pythagoras theorem, Transformation, Solid Geometry II, and statistics. The mathematical values among students in the experimental group improved significantly after the PBL strategy intervention. The PBL strategy usage was useful and effective to guide students' mathematical values compared with the normal instructional activities. The PBL strategy played a vital role in enhancing students' participation and interest in discovering mathematical problems instead of developing students' mathematical values. The implementation of the PBL strategy in mathematical learning activities allowed students to engage in deeper mathematical values.

\section{REFERENCES}

[1] Amalia, E., Surya, E. \& Syahputra, E. (2017). The effectiveness of using problem-based learning (PBL) in mathematics problem-solving ability for junior high school students. International Journal of Advance Research and Innovative Ideas in Education. 3(2), 3402 - 3406

[2] Bakar, K.A., Ayub, A.F.M., Mahmud, R. (2015). Effects of GeoGebra on students' Mathematics performance. In Abd Majid (Eds) Proceedings of the IEEE 7th International Conference on Research and Education in Mathematics:
Empowering Mathematical Sciences through Research and Education (pp. 180-183). Serdang, Universiti Putra Malaysia: Institute For Mathematical Research DOI: 10.1109/ICREM.2015.7357049

[3] Barrows, H. S. (1986). A taxonomy of problem-based learning methods. Medical Education, 20, 481-486.

[4] Bishop, A. J. (1988). Mathematical enculturation: A cultural perspective on mathematics education. Dordrecht: Kluwer Academic Publishers.

[5] Bishop, A., Clarkson, P., FitzSimons, G., and Seah, W.T. (2000). Why Study Values in Mathematics Teaching: Contextualising the VAMP Project.

[6] Brown, R. (2001). Educational values and summative assessment a view across three educational systems. Paper presented at the Annual Conference of the Australian Association for Research in Education, Fremantle, Australia.

[7] Chin C., Leu Y.C., Lin F.L. (2001) Pedagogical values, mathematics teaching, and teacher education: Case studies of two experienced teachers. In: Lin FL., Cooney T.J. (eds) Making Sense of Mathematics Teacher Education. Springer, Dordrecht. https://doi.org/10.1007/978-94-010-0828-0_12.

[8] Cohen, J. (1988). Statistical power analysis for the behavioral sciences $\left(2^{\text {nd }}\right.$. ed.). Hillsdale, NJ: Lawrence Earlbaum Associates.

[9] Goodman, R. J. B. (2010). Problem-Based Learning: Merging of economics and mathematics. Journal of Economic Finance, 34, 477-483.

[10] Graaff, E. de and Kolmos, A. (2003). Characteristics of problem-based learning, International Journal of Engineering Education, 5, 657-662.

[11] Hung, W. (2016). All PBL starts here: The problem. Interdisciplinary Journal of Problem-Based Learning, 10 (2). https://doi.org/10.7771/1541-5015.1604.

[12] Ibrahim, N.N. Ayub, A.F.M. Yunus, A.S.M. Mahmud, R. Bakar, K.A. (2019). Effects of higher-order thinking module approach on pupils' performance at primary rural school. Malaysian Journal of Mathematical Sciences, 13 (2), 211-229

[13] Ibrahim, N. N., Mohd Ayub, A. F., Md. Yunus, A. S., Gopal, K., \& Salim, N.R (2019). Effects of HOTS-based Module Approach on Pupil's Errors in the Topic of Measurement and Geometry in Urban and Rural Schools. Universal Journal of Educational Research. 7(11), 2519-2535.

[14] Jones, M. (1999) Spiritual, moral, social and cultural education. Exploring values in the curriculum. David Fulton Publishers, London.

[15] Lim, C. S., \& Ernest, P. (1997). Values in mathematics education: What is planned and what is espoused? Paper presented at the Conference of the British Society for Research into Learning Mathematics, Nottingham, U.K.

[16] Mokhtar, M. Z., Tarmizi, R. A., Ayub, A. F. M., and Tarmizi, M. A. A. (2010). Enhancing calculus learning engineering students through problem-based learning. WSEAS transactions on Advances in Engineering Education, 7(8), $255-264$.

[17] Nasir, N.S., Hand, V., \& Taylor, E.V. (2008). Culture and 
mathematics in school: Boundaries between "cultural" and "domain" knowledge in the mathematics classroom and beyond. Review of Research in Education, 32, 187-240.

[18] Ramli, F., Ayub, A.F.M, \& Salim, R., (2018). Effectiveness of problem-based learning strategy towards solving higher and lower order questions among secondary school students. In Maidinsah H., Sharif S.R., Rahman W.E.Z.W.A., Akbarally A.B., Mohamed M., Mohamad D., Jaffar M.M (ed.). AIP Conference Proceedings: 25th National Symposium on Mathematical Sciences: Mathematical Sciences as the Core of Intellectual Excellence, SKSM 2017 (pg. 1- 7). Pahang, Malaysia: Universiti Teknologi MARA.

[19] Saha, R.A., Ayub, A.F.M., Tarmizi, R.A. (2010). The effects of GeoGebra on mathematics achievement: Enlightening Coordinate Geometry learning. Procedia - Social and Behavioral Sciences, 8, 686-693

[20] Seah, W. T. \& Bishop, A.J. (2000). Values in mathematics textbooks: A view through the Australasian regions. Paper Presented at the Annual Meeting of the American Educational Research Association, New Orleans, LA.

[21] Seah, W. T. \& Bishop, A.J. (2002). Values, Mathematics and
Society: Making The Connections. In C. Vale \& J. Roumeliotis \& J. Horwood (Eds.), Valuing mathematics in society (pp. 105-113). Brunswick, Australia: Mathematical Association of Victoria.

[22] Seah, W. T. (2002). Exploring teacher clarification of values relating to mathematics education. In C. Vale, J. Roumeliotis \& J. Horwood (Eds.), Valuing mathematics in society (pp. 93-104). Brunswick, Australia: Mathematical Association of Victoria.

[23] Seah, W. T., Bishop, A. J., FitzSimons, G. E., \& Clarkson, P. C. (2001). Exploring issues of control over values teaching in the mathematics classroom. Paper presented at the 2001 Annual Conference of the Australian Association for Research.

[24] Seah, W.T. (2013) Values in the mathematics classroom: Supporting cognitive and affective pedagogical ideas, Gazi Journal of Education, 1, 45-65.

[25] Swadener M., Soedjadi R. (1988). Values, Mathematics Education, and the Task of Developing Pupils' Personalities: An Indonesian Perspective. In: Bishop A.J. (eds). Mathematics Education and Culture. Springer, Dordrecht. https://doi.org/10.1007/978-94-017-2209-4_5. 